\title{
A Beacon in the Dark: Canakinumab. A New Therapeutic Perspective in Chronic Tophaceous Gout
}

Daniela Marotto - Antonella De Santis - Donatella Chessa •

Davide Firinu $\cdot$ Stefano Del Giacco

Received: December 14, 2017 / Published online: March 9, 2018

(C) The Author(s) 2018. This article is an open access publication

\section{ABSTRACT}

Gout is the most common form of arthritis in adults. It is often associated with other comorbidities, which contraindicate the use of conventional therapies. The discovery of the role of interleukin-1 $\beta$ (IL-1 $\beta$ ) in orchestrating the monosodium urate crystal-induced inflammatory response offered new therapeutic prospects to refractory patients, or to those in whom standard therapies are contraindicated. This paper describes a clinical case of a 65-year-old man with chronic tophaceous gouty arthropathy and subintrant flares, who had comorbidities contraindicating the use of conventional gout therapies-to which he did not respondwho was treated with canakinumab, a monoclonal selective inhibitor of IL-1 $\beta$. The patient

Enhanced content To view enhanced content for this article go to https://doi.org/10.6084/m9.figshare.59155 15.

\section{Marotto $(\square)$}

Rheumatology, Department of Medical Sciences and Public Health, Assl Olbia, Olbia, Italy

e-mail: daniela.marotto@tiscali.it;

Dmarotto@aslolbia.it

A. De Santis - D. Chessa

Department of Medical Sciences and Public Health, Assl Olbia, Olbia, Italy

D. Firinu $\cdot$ S. Del Giacco

Department of Medical Sciences and Public Health, University of Cagliari, Cagliari, Italy reported a gradual, rapid, and significant reduction in pain, with a response observed within $12 \mathrm{~h}$ of the administration of the drug. Consistent with previous clinical studies, canakinumab appeared to be a viable, safe, and effective alternative to conventional therapies in this patient with gout who had limited therapeutic options.

Funding: Novartis Farma, Italy

Keywords: Canakinumab; Chronic tophaceous gout; Interleukin-1 $\beta$; Refractory gouty arthropathy; Treatment options

\section{INTRODUCTION}

Known since ancient times, gout is the most common form of arthritis among the adult population, with a prevalence of $4 \%$ in the USA and $0.46 \%$ in Italy; it affects approximately 8.3 million people in America alone $[1,2]$. The economic burden of gout is significant, not only because epidemiological data show an increase in its prevalence and incidence worldwide but also because it has a high level of disability and mortality [3].

Gout is caused by the deposit of monosodium urate crystals inside the joints and tissues. The drugs conventionally used for the treatment of acute gout are non-steroidal anti- 
inflammatory drugs (NSAIDs), including cyclooxygenase (COX)-2 inhibitors, colchicine, and corticosteroids [4]. However, an increasing number of patients with gout are intolerant or refractory, or have contraindications, to the use of conventional drugs.

Recently, studies have highlighted the key role of interleukin-1 $\beta$ (IL-1 $\beta$ ) in the inflammatory process related to the tissue and articular deposit of monosodium urate crystals in patients with gout [5-9]. According to this theory, at the start of a bout of acute gout, monosodium urate crystals activate inflammasome NLRP3, leading to the release of IL-1 $\beta$ and consequently an inflammatory response, with vasodilation and recruitment of immune cells, preferentially neutrophils, in the site of the crystal deposit. Based on the identification of the role of IL-1 $\beta$ in gout, development of drugs that inhibit IL-1 signal transduction was initiated, expanding the small number of therapeutic options available for the management of the acute attack in the patient with gouty arthritis.

Among currently available IL-1 inhibitors are anakinra, an IL-1 receptor human antagonist, rilonacept, an inhibitor of both IL- $1 \beta$ and IL- $1 \alpha$, and canakinumab, a fully human monoclonal antibody selectively inhibiting IL-1 $\beta$. Several publications have demonstrated the efficacy of these drugs in patients with gout for whom conventional therapies are contraindicated or ineffective [10-15].

Reported here is the case of a patient with chronic tophaceous gout refractory to conventional therapies, to the use of which he also presented contraindications, who responded to treatment with canakinumab.

\section{CASE REPORT}

In October 2016, a 65-year-old male patient, who had been hospitalized for acute heart failure, was referred to our operative unit (OU) for a consultation by the medicine OU of the same hospital for a severe acute attack of gout. He had a history of type 2 diabetes mellitus, arterial hypertension, ischemic dilated cardiomyopathy, and chronic atrial fibrillation. He had also experienced a previous myocardial infarction, had chronic grade III renal insufficiency for the last 3 years, was obese, and was diagnosed with gouty arthropathy in 2011. Since his diagnosis of gout the patient had experienced over 15 acute attacks, and was treated with colchicine, highdose steroids, NSAIDs, and allopurinol (replaced in 2016 with febuxostat). His family history was negative for gout, and there was a personal history of high dietary purine intake and previous smoking and alcohol abuse.

At the time of referral, the patient had swelling of multiple joints, including his right knee, wrists, and the metacarpophalangeal (MCP) and interphalangeal (IP) joints of his hands, feet, and ankles. In the days prior to hospitalization, he had progressive clinical worsening of his gout, which he treated with NSAIDs, colchicine (up to $3 \mathrm{mg} /$ day), and highdose steroids without any benefit. He presented at the emergency room of our hospital where, over several days additional boluses of steroids were administered without clinical response, but with a worsening of dyspnea, fatigue, poor control of blood pressure, and onset of swelling. At admission, he was receiving the following therapies: bisoprolol $7.5 \mathrm{mg}$ /day $(5+2.5 \mathrm{mg})$, colchicine $1 \mathrm{mg}$ twice daily, rivaroxaban $15 \mathrm{mg}$ /day, prednisone $25 \mathrm{mg} /$ day, tramadol as needed, pantoprazole $40 \mathrm{mg} / \mathrm{day}$, febuxostat $80 \mathrm{mg} /$ day, and olmesartan $20 \mathrm{mg} /$ day.

Upon physical examination, the patient was in severe pain, numerous tophaceous nodules were observed bilaterally on the hands, feet, elbows, and knees (Figs. 1a, 2), and symmetric arthritis of the feet, right knee, and right hand was present, with the overlying skin warm and erythematous. Walking was impossible.

Blood tests revealed elevated uric acid, blood urea nitrogen, blood count, serum creatinine, erythrocyte sedimentation rate, and C-reactive protein (Table 1), while culture tests (blood culture, urinoculture, stool test, synovial fluid culture), tests for antinuclear antibodies, antidouble stranded DNA, anti-citrullinated protein antibody, extractable nuclear antigen 
A
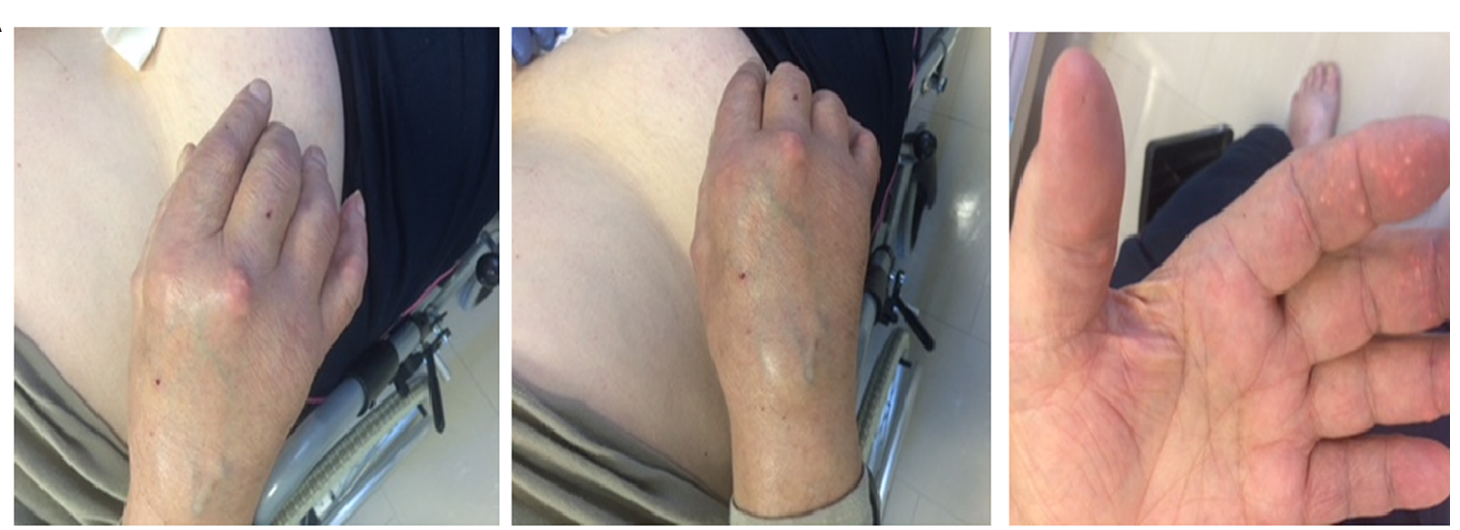

B
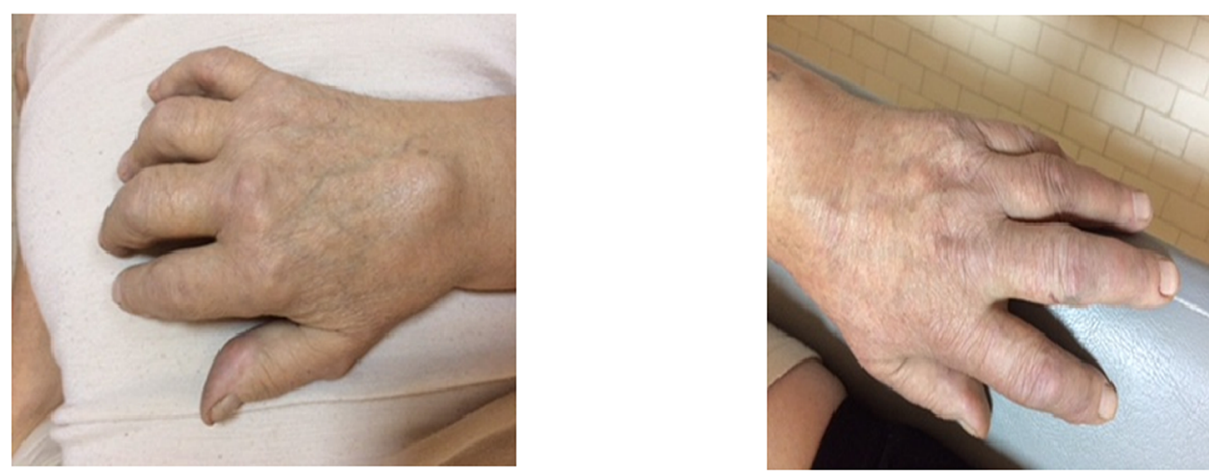

C
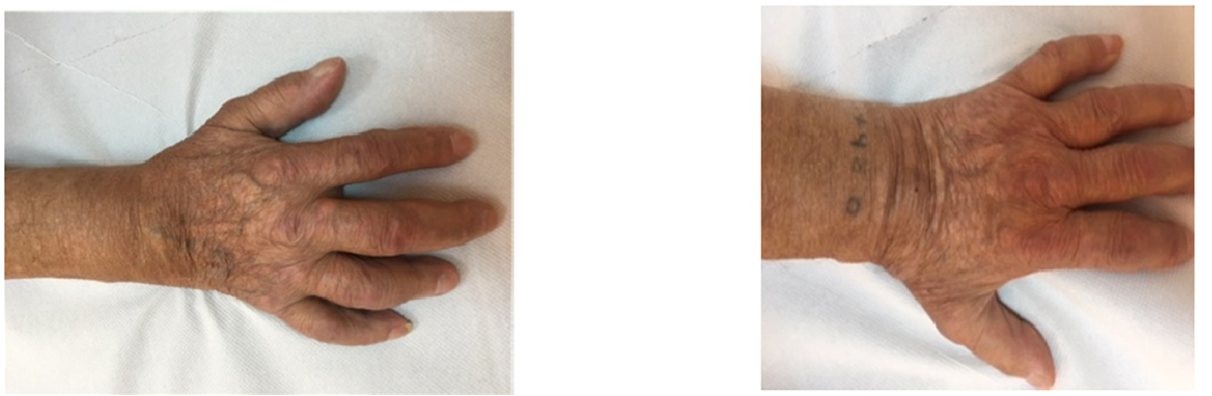

Fig. 1 Joint swelling and tophi in the right and left hands $\mathbf{a}$ at the start of treatment, $\mathbf{b}$ at day 14 , and $\mathbf{c}$ at day 30

antibodies, rheumatoid factor, prostate-specific antigen, hepatitis B and hepatitis C markers, and his thyroid profile were all negative. The examination of the right knee synovial fluid showed the presence of monosodium urate crystals, with marked leukocytosis.

An X-ray of the hands revealed multiple asymmetrical thickening of periarticular soft tissue, with increased nubecula-type density, erosions of the interphalangeal joints, cystic lesions and initial reduction of the corresponding articular rimae, while a chest X-ray was normal. Echocardiography showed widely dilated left ventricular hypokinetic LV and an ejection fraction of $20 \%$ while electrocardiography (ECG) revealed full atrial fibrillation arrhythmia, an average heart rate of $95 \mathrm{bpm}$, and left bundle branch block.

Considering the failure to respond to conventional gout therapies, to which the patient by now had proved to be refractory, as well as the contraindications to their use due to his comorbidities, canakinumab was administered subcutaneously, in a single dose of $150 \mathrm{mg}$. 

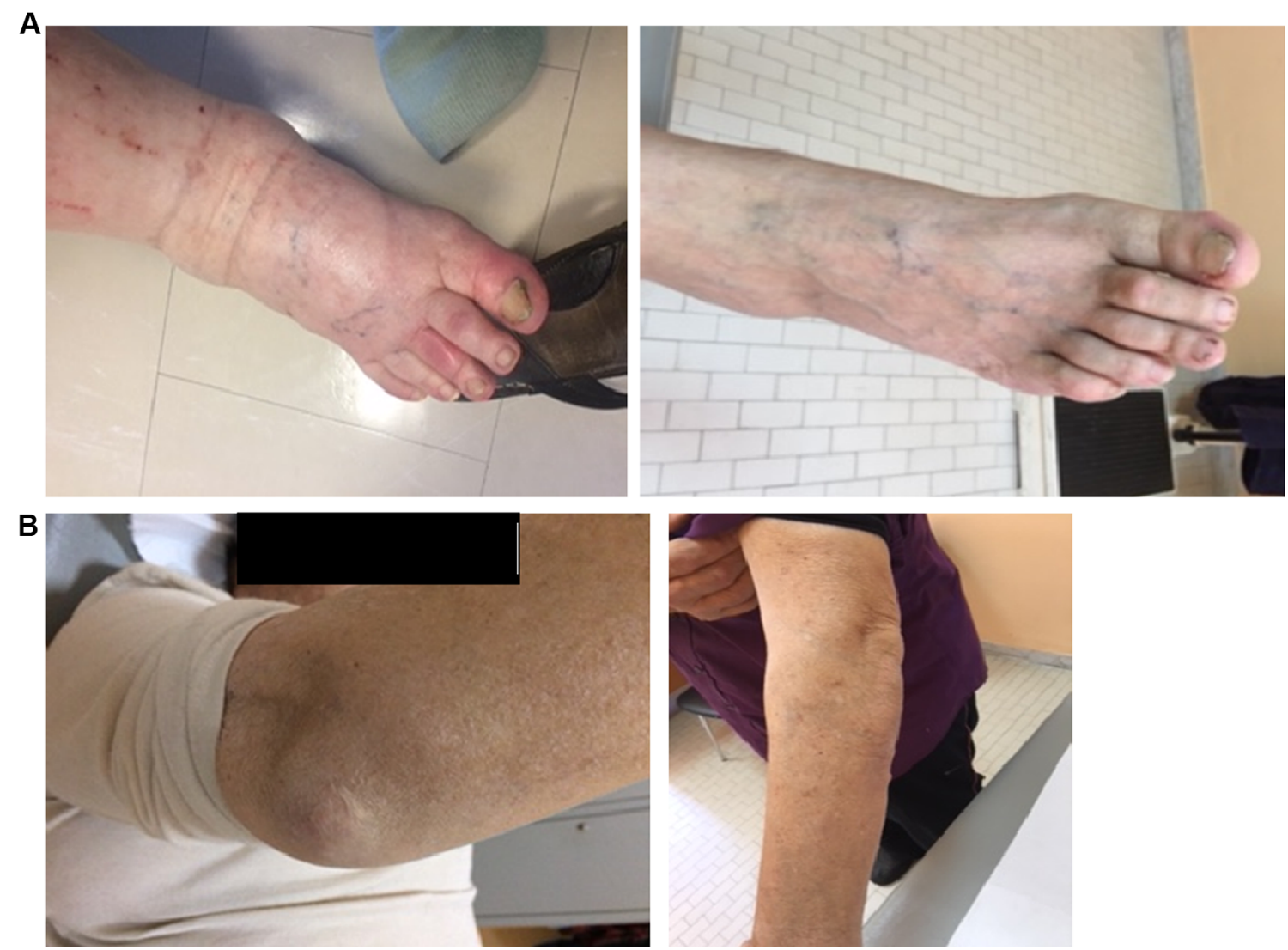

Fig. 2 Change in joint swelling a from baseline to day 30 in the right foot, $\mathbf{b}$ from day 14 to day 30 in the right elbow

The patient reported a gradual, rapid, and significant reduction in pain (calculated using a 0-100 VAS scale), which was apparent $12 \mathrm{~h}$ after the administration of the drug (Fig. 3a). After $24 \mathrm{~h}$, the patient was able to walk independently, without any help or support. Over the next few days there was a progressive improvement in the patient's daily activities, as assessed by the Barthel Index of Activities of Daily Living and the Lawton-Brody Instrumental Activities of Daily Living Scale, which improved from 30 to 100 and from $2 / 5$ to $5 / 5$ at 4 weeks after treatment. In parallel with the reduction of pain, the dose of his steroid (prednisone) was gradually scaled down, until its complete suspension, after 22 days (Fig. 3b). Blood tests carried out 10 days after the administration showed improvement of all parameters previously elevated (Table 2).
Fourteen days after receiving canakinumab, the patient was discharged from hospital. At this time, the patient had a marked improvement in his cardiac and renal function. Echocardiography revealed post-ischemic dilated hypokinetic cardiomyopathy, severe dilation of the left ventricle, moderate reduction in global kinetics, moderate-severe diastolic dysfunction, biatrial enlargement (severe in left atrium), mild pulmonary hypertension, mild mitral insufficiency, ectasia of the aortic root and ascending aorta, and an injection fraction of $40 \%$.

The patient was discharged with instructions to take tramadol three times per day as needed, which he did not (as reported at the follow-up visits made on days 30, 45, 60, and 90); the patient also reported no new acute attacks or adverse events. 
Table 1 Characteristics and demographics of patient at hospital admission

\begin{tabular}{ll}
\hline Parameter & Value \\
\hline Age, years & 65 \\
Height, cm & 170 \\
Weight, kg & 107 \\
BMI, kg/m ${ }^{2}$ & 37 \\
Ejection fraction, \% & 20 \\
Heart rate, bpm & 95 \\
Blood test results & \\
Uric acid, mg/dL & 10 \\
ESR, mm/h & 96 \\
CRP, mg/dL & 28.99 \\
WBC, $/ \mathrm{mm}{ }^{3}$ & 14,600 \\
Neutrophils, \% & 87.9 \\
Lymphocytes, \% & 4.3 \\
Creatinine, $\mu \mathrm{mol} / \mathrm{L}$ & 176 \\
Creatinine clearance ${ }^{\mathrm{a}}, \mathrm{mL} / \mathrm{min}$ & 56.47 \\
Calcium, mg/dL & 7.9 \\
Azotemia, mg/dL & 78 \\
Glucose, mg/dL & 142 \\
Albumin, g/dL & 2.4 \\
AST, U/L & 9 \\
ALT, U/L & 7 \\
GGT, U/L & 44 \\
\hline
\end{tabular}

${ }^{a}$ Assessed using the Cockcroft-Gault equation $A L T$ alanine aminotransferase, $A S T$ aspartate aminotransferase, $B M I$ body mass index, $C R P C$-reactive protein, $E S R$ erythrocyte sedimentation rate, $G G T$ gamma-glutamyl transferase

Four weeks after the administration of canakinumab, he presented for a follow-up, where the number and size of tophi observed at hospitalization were reduced (Figs. 1c and 2).

Informed consent was obtained from the patient for his information to be used in this case report.

\section{DISCUSSION}

To date, canakinumab is the only drug to have obtained approval from the Italian Medicines Agency (AIFA) for the symptomatic treatment of adult patients with gouty arthritis. Canakinumab is approved by the European Medicines Agency for the treatment of adult patients with frequent attacks of gouty arthritis refractory to NSAIDs, colchicine, and repeated courses of corticosteroids $[16,17]$. The efficacy of canakinumab was investigated in two phase 2 variabledose studies $[11,12]$ and in two phase 3 , randomized, multicenter, double-blind studies $(\beta$ RELIEVED and $\beta$-RELIEVED-II) [10], which showed that a single dose of canakinumab (150 mg) can control new exacerbations and causes a rapid reduction of the pain and inflammatory state in patients with gout who have contraindications, intolerance, or non-responsiveness to colchicine and/or to NSAIDs. Long-term follow-up studies with canakinumab have also reported similar efficacy [18]. Post hoc analysis of the phase 3 studies to determine the safety and efficacy of canakinumab in a subgroup of patients refractory to NSAIDs and colchicine reported similar results to the main studies [19]. Canakinumab is also reported to improve health-related quality of life in patients with difficult-to-treat gouty arthritis [20]. The safety profile of canakinumab from the phase 3 studies showed that the treatment is well tolerated and the main adverse events reported with treatment include infections, hypertension, headache, and back pain [10]. However, canakinumab is not approved by the US Food and Drug Administration for the treatment of gouty arthritis because of concerns regarding its safety, particularly related to the incidence of infections and hypertension with its use [21].

Despite the presence of multiple comorbidities (diabetes, hypertension, heart failure) contraindicating the use of conventional therapies, the baseline characteristics of the patient in the present case report were globally similar to the patients enrolled in the phase 3 studies with canakinumab [10]. Our case, which appears to be the first in Italy in which a patient with gouty arthropathy refractory to conventional 

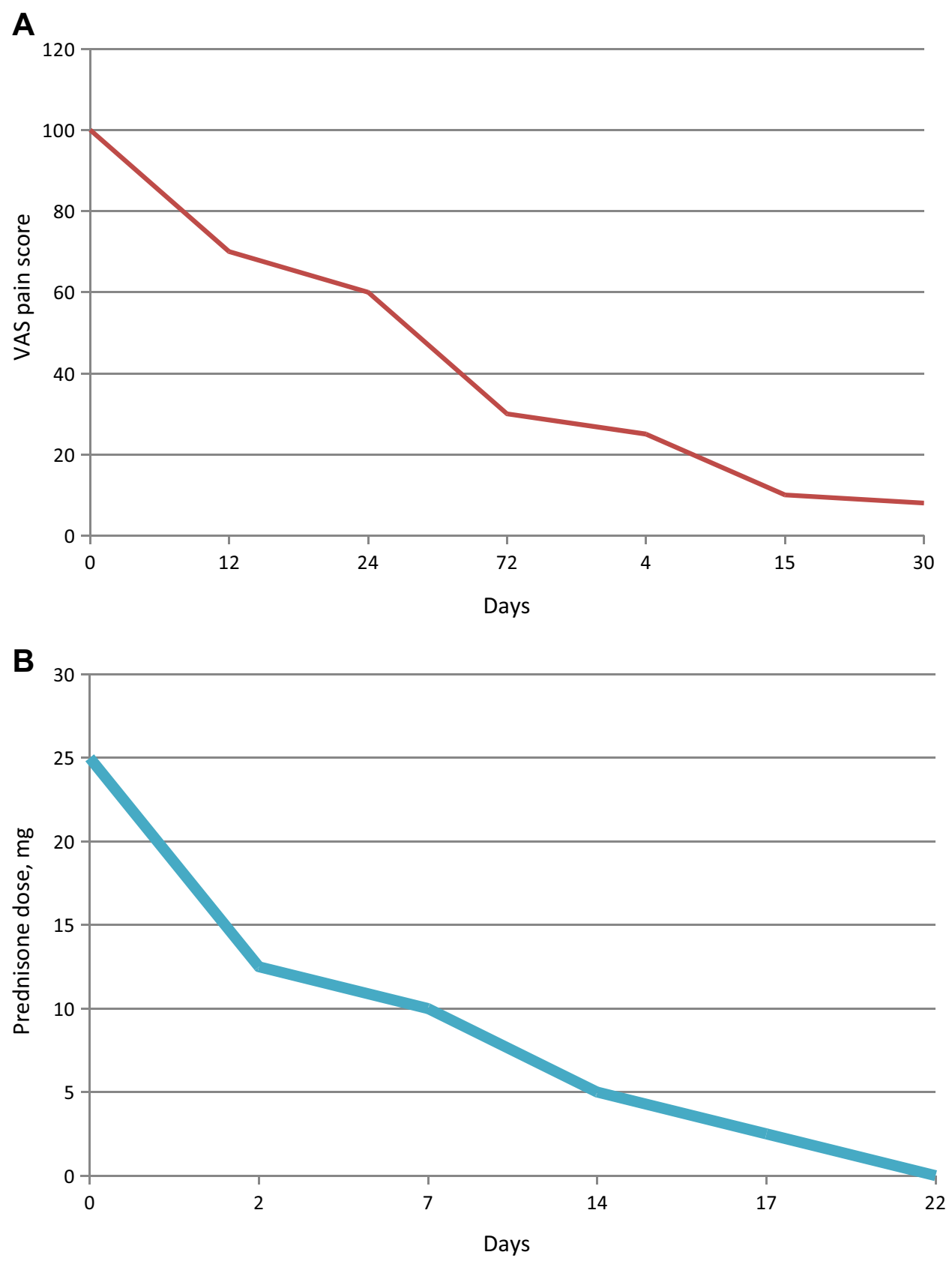

Fig. 3 Change in a visual analogue scale (VAS) pain score and $\mathbf{b}$ dose of steroid (prednisone) over time after administration of canakinumab

therapies was treated with canakinumab, supports the findings of the clinical trials by demonstrating how a single dose of canakinumab $150 \mathrm{mg}$ results in a rapid and significant improvement of the pain, the inflammatory state, and thus the patient's quality of life. A single dose of canakinumab (150 mg) also seemed to reduce the number and size of tophi in the patient, and further studies are required to confirm this effect. 
Table 2 Blood test results at 10 days

\begin{tabular}{ll}
\hline Blood test & $\boldsymbol{N}=\mathbf{1}$ \\
\hline Uric acid, mg/dL & 8.4 \\
ESR, mm/h & 22 \\
CRP, mg/dL & 1.09 \\
White blood cells, $\times 10^{9} / \mathrm{L}$ & 6.90 \\
Neutrophils, \% & 57.4 \\
Lymphocytes, \% & 28.7 \\
Creatinine & 1.8 \\
\hline
\end{tabular}

$C R P$ C-reactive protein, ESR erythrocyte sedimentation rate

\section{ACKNOWLEDGEMENTS}

Funding. The study and article processing charges were funded by Novartis Farma, Italy. All authors had full access to all of the data in this study and take complete responsibility for the integrity of the data and accuracy of the data analysis.

Authorship. The named authors meet the International Committee of Medical Journal Editors (ICMJE) criteria for authorship for this manuscript, take responsibility for the integrity of the work as a whole, and have given final approval for the version to be published.

Medical Writing and/or Editorial Assistance. We would like to thank Simone Boniface and Nishad Parkar, PhD, of Springer Healthcare Communications who edited the English of the manuscript prior to submission and helped with the peer review comments post submission, respectively. This medical writing support was funded by Novartis Farma, Italy.

Disclosures. Daniela Marotto, Antonella De Santis, Donatella Chessa, Davide Firinu, and Stefano Del Giacco have nothing to disclose.

Compliance with Ethics Guidelines. Informed consent was obtained from the patient for his information to be used in this case report.
Data Availability. Data sharing is not applicable to this article as no datasets were generated or analyzed during the current study.

Open Access. This article is distributed under the terms of the Creative Commons Attribution-NonCommercial 4.0 International License (http://creativecommons.org/licenses/ by-nc/4.0/), which permits any noncommercial use, distribution, and reproduction in any medium, provided you give appropriate credit to the original author(s) and the source, provide a link to the Creative Commons license, and indicate if changes were made.

\section{REFERENCES}

1. Kuo CF, Grainge MJ, Zhang W, Doherty M. Global epidemiology of gout: prevalence, incidence and risk factors. Nat Rev Rheumatol. 2015;11:649-62.

2. Zhu Y, Pandya BJ, Choi HK. Prevalence of gout and hyperuricemia in the US general population: the National Health and Nutrition Examination Survey 2007-2008. Arthritis Rheum. 2011;63:3136-41.

3. Shields GE, Beard SM. A systematic review of the economic and humanistic burden of gout. Pharmacoeconomics. 2015;33:1029-47.

4. Richette P, Doherty M, Pascual E, et al. 2016 updated EULAR evidence-based recommendations for the management of gout. Ann Rheum Dis. 2017;76:29-42.

5. Akahoshi T, Murakami Y, Kitasato H. Recent advances in crystal-induced acute inflammation. Curr Opin Rheumatol. 2007;19:146-50.

6. Di Giovine FS, Malawista SE, Nuki G, Duff GW. Interleukin 1 (IL 1) as a mediator of crystal arthritis. Stimulation of $\mathrm{T}$ cell and synovial fibroblast mitogenesis by urate crystal-induced IL 1 . J Immunol. 1987;138:3213-8.

7. Martinon F, Pétrilli V, Mayor A, et al. Gout-associated uric acid crystals activate the NALP3 inflammasome. Nature. 2006;440:237-41.

8. Murakami Y, Akahoshi T, Hayashi I, et al. Inhibition of monosodium urate monohydrate crystalinduced acute inflammation by retrovirally transfected prostaglandin D synthase. Arthritis Rheum. 2003;48:2931-41. 
9. Roberge CJ, Grassi J, De Medicis R, et al. Crystalneutrophil interactions lead to interleukin-1 synthesis. Agents Actions. 1991;34:38-41.

10. Schlesinger N, Alten RE, Bardin T, et al. Canakinumab for acute gouty arthritis in patients with limited treatment options: results from two randomised, multicentre, active-controlled, double-blind trials and their initial extensions. Ann Rheum Dis. 2012;71:1839-48.

11. Schlesinger N, Mysler E, Lin HY, et al. Canakinumab reduces the risk of acute gouty arthritis flares during initiation of allopurinol treatment: results of a double-blind, randomised study. Ann Rheum Dis. 2011;70:1264-71.

12. So A, Meulemeester De, Pikhlak A, et al. Canakinumab for the treatment of acute flares in difficultto-treat gouty arthritis: results of a multicenter, phase II, dose-ranging study. Arthritis Rheum. 2010;62:3064-76.

13. Van Wabeke J, Dhondt E, Peene I, Piette Y. Anakinra in resistant gout: a case report. Acta Clin Belg. 2017;72:293-5.

14. Thueringer JT, Doll NK, Gertner E. Anakinra for the treatment of acute severe gout in critically ill patients. Semin Arthritis Rheum. 2015;45:81-5.

15. Sundy JS, Schumacher HR, Kivitz A, et al. Rilonacept for gout flare prevention in patients receiving uric acid-lowering therapy: results of RESURGE, a phase III, international safety study. J Rheumatol. 2014;41:1703-11.
16. Novartis Pharma GmbH. Canakinumab Summary of product characteristics. 2014. http://www.ema. europa.eu/docs/en_GB/document_library/EPAR_-_ Product_Information/human/001109/ WC500031680.pdf. Accessed 13 Feb 2018

17. Pazár Maldonado B, So A. Canakinumab for the treatment of gout. Int $\mathrm{J}$ Clin Rheumatol. 2011;6:605-15.

18. Schlesinger N, Alten R, Bardin T, et al. Canakinumab in frequently flaring gouty arthritis patients, contraindicated, intolerant or unresponsive to nonsteroidal anti-inflammatory drugs and/or colchicine: safety and efficacy results from long term follow-up. Arthritis Rheum. 2014;66:S66-7.

19. Bardin T, So A, Alten R, et al. Efficacy and safety of canakinumab vs triamcinolone acetonide in patients with gouty arthritis unable to use nonsteroidal anti-inflammatory drugs and colchicine, and on stable urate lowering therapy (ULT) or unable to use ULT. Arthritis Rheum. 2012;64:S811-2.

20. Schlesinger N, De Meulemeester M, Pikhlak A, et al. Canakinumab relieves symptoms of acute flares and improves health-related quality of life in patients with difficult-to-treat gouty arthritis by suppressing inflammation: results of a randomized, dose-ranging study. Arthritis Res Ther. 2011;13:R53.

21. Lowry F. FDA panel says no to canakinumab for gout attacks. 2011. https://www.medscape.com/ viewarticle/745076. Accessed 13 Feb 2018. 